\title{
Creatividad y modelos de enseñanza a través de la expresión corporal, en el ámbito deeducación secundaria obligatoria Musical creativity through body expression, in the field of compulsory secondary education
}

\author{
Sara Cuevas Romero \\ Universidad de Sevilla
}

\begin{abstract}
Resumen: Presentamos una investigación, basada en la creación como desarrollo de un proceso de aprendizaje entre el alumnado de primero de Educación Secundaria Obligatoria, que parte de los cuatro parámetros del sonido, y la búsqueda de recursos musicales y expresivos, donde convergen la composición, la interpretación y la expresión corporal. Desde el punto de vista metodológico, nos encontramos ante una investigación empírica (experimental), basada en la recogida de datos de una realidad educativa, orientada a la mejora de la práctica docente. Los resultados en esta investigación, se obtienen tras realizar un análisis estadístico de los datos obtenidos sobre el nivel de inventiva y de comunicación, para comprobar si existen diferencias significativas en el desarrollo creativo del alumnado de $1^{\circ} \mathrm{ESO}$, tras la utilización de dos modelos de enseñanza diferentes (modelo tradicional y modelo creativo).
\end{abstract}

Palabras clave: Expresión corporal, creación musical, creatividad, Educación Secundaria Obligatoria, modelo de enseñanza tradicional, modelo de enseñanza creativo.

\begin{abstract}
We present an investigation, based on the creation and development of a learning process among students in Secondary Education first, that some of the four parameters of the sound, and the pursuit of musical resources and expressive, which converge composition, performance and body language. From the methodological point of view, we are faced with an empirical (experimental), based on the collection of data in an educational reality, aimed at improving teaching practice. The results in this research, are obtained after statistical analysis of the data obtained on the level of inventiveness and communication, to check if there are significant differences in the creative development of students in 1st ESO, after the use of two models of teaching different (traditional model and creative model).
\end{abstract}

Key words: Body language, musical creation, creativity, Secondary Education, traditional teaching model, creative teaching model.

\section{Introducción}

En este artículo nos hemos centrado en la importancia que adquiere el desarrollo de la creatividad en el alumnado de Educación Secundaria Obligatoria, a través de la expresión corporal en la creación musical.

Teniendo en cuenta que es, el sistema educativo, el que nos influye en la forma en la que enseñamos y en el estilo de enseñanza que utilizamos en nuestra práctica diaria en las aulas, autores como Rivas (2008) destaca que debemos preparar a nuestros alumnos para aprender en todas las áreas y disciplinas de conocimiento. Siguiendo a este autor, «las personas adquieren, pues, mediante el aprendizaje, distintos tipos de capacidades, en las que puede incluirse congruentemente la multiplicidad de lo que aprenden», como es el desarrollo de su creatividad.

Por otro lado, autores como Gervilla (1986), ya detectaron en décadas anteriores que «la cultura y los sistemas de enseñanza, la sociedad y los individuos necesitan hoy, reclaman, la formación para la creatividad.» Esta afirmación se contempla actualmente en el desarrollo legislativo vigente en el currículum de la materia de música en Educación Secundaria Obligatoria.

La investigación que aquí presentamos, desarrollada a lo largo del curso académico 2010/2011, se inscribe en el marco legal actual, que es en el que nos hemos centrado y presentamos a continuación.

En la actualidad, el currículum de Educación Secundaria Obligatoria, se rige por la Ley Orgánica 2/2006, de 3 de mayo, de Educación, por el que se establecen las enseñanzas mínimas correspondientes a la ESO; y el Real Decreto 1631/2006, de 29 de diciembre, es donde se establecen las enseñanzas mínimas correspondientes a la Educación Secundaria Obligatoria, en relación a uno de los objetivos de esta etapa.

EnEducación Secundaria, atendiendo el desarrollo de la percepción del alumno, destacamos que se deben desarrollar actividades de interpretación y creación musical; y a través de la expresión, el desarrollo de todas aquellas capacidades vinculadas también con la interpretación y la creación. Mediante el desarrollo de las capacidades vinculadas a la ex-

Fecha recepción: 28-10-13- Fecha envío revisores: 28-10-13- Fecha de aceptación: 12-11-13 Sara Cuevas Romero

C/Moscú, 12.

CP 11405. Jerez de la Fra. (Cádiz)

saracuevasr@hotmail.com presión, se pretende facilitar el logro de un dominio básico de las técnicas requeridas para el canto y la interpretación instrumental, además de ajustes rítmicos y motores implícitos en el movimiento y la danza, propios de la expresión corporal.

Algunos autores como Muñoz (2007), dan mucha importancia al desarrollo de la expresión a lo largo de la educación obligatoria. Para este autor, la finalidad que persigue la expresión musical no es otra que, «ofrecer al alumnado un medio para la expresión, la comunicacióny la vivencia musical», en el que desde nuestro punto de vista adquiere mucha importancia fomentar el desarrollo creativo a través de la expresión corporal.

Atendiendo a la importancia que la educación tiene en la promoción de aprendizajes creativos, citamos a Pastor (1998), el cual afirma que «la educación desempeña un papel decisivo en el desarrollo de la creatividad y viceversa», además de contemplar la necesidad de crear que tiene el ser humano, aspecto que no podemos obviar en el desarrollo integral de nuestro alumnado.

Por otro lado, destacamos que el desarrollo didáctico de una creación musical considerada como proceso de aprendizaje que promueve la creatividad, además de actitudes críticas. Para Kanellopoulos (2012), la esencia de la creación se encuentra en la composición.

Esta investigación que presentamos, ha sido desarrollada durante tres meses, en $1^{\circ} \mathrm{ESO}$, consistiendo en la creación de una obra musical en grupo, a partir de los propios conocimientos musicales de los alumnos y los cuatro parámetros del sonido.

Desde un primer momento, los alumnos saben que todos los miembros del grupo deben dar sus opiniones a los demás, sobre los instrumentos, las voces, los textos, los movimientos, los recursos o los conceptos musicales que van a utilizar, contando con total libertad en la utilización de sus aportaciones, es decir, el manejo y empleo de los conceptos musicales y corporales que utilicen no tiene que ser convencionales, deben experimentar.

A lo largo del proceso de enseñanza - aprendizaje, en cada una de las sesiones, se han promovido conocimientos teóricos y prácticos, trabajando de manera secuenciada los cuatro parámetros del sonido y el movimiento, favoreciendo en cada una de ellas el mayor desarrollo creativo posible.

Las decisiones metodológicas que hemos tenido presentes a lo largo de esta experiencia, en base a los principios anteriormente expuestos, 
siguiendo a Cuevas (2011), son los siguientes aspectos:

- Se debe fomentar la participación del alumnado en el grupo como principales protagonistas de su propio aprendizaje.

- Se debe estimular el desarrollo creativo del alumnado a lo largo del proceso de enseñanza-aprendizaje a través de la música y el movimiento creativo.

- Se debe fomentar la utilización de movimientos creativos a través de la música.

- Se debe orientar el movimiento creativo de los alumnos a través de lamúsica.

- Se deben potenciar habilidades reflexivas y críticas a través de la música y el movimiento creativo.

En esta investigación partimos del planteamiento de que nuestro alumnado puede desarrollarse creativamente, y concretamente en nuestro estudio, a través de la expresión corporal, siempre que a lo largo de su aprendizaje se fomenten estas capacidades. Justificando este planteamiento inicial del que partimos vamos a tratar de dar respuesta a esta postura en la que nos posicionamos, llevándolo a nuestra práctica educativa, para averiguar si existe o no relación entre el desarrollo creativo del alumnado de $1^{\circ}$ y la utilización de dos modelos de enseñanza diferentes, a través de la expresión corporal. Para ello, trabajaremos con nuestro alumnado de ESO, mediante un modelo de enseñanza tradicional y un modelo creativo.

\section{La expresión corporal, a través de la creación musical}

La música, en las actividades desarrolladas a través de la expresión corporal, tiene como finalidad, ser un medio para desarrollar la creatividad, por lo que consideramos importante favorecer aprendizajes en los que la creación, tanto musical como corporal, estén presentes.

La expresión corporal en Educación Secundaria Obligatoria, promueve el desarrollo de capacidades, destrezas y habilidades motrices, cognitivas y afectivas, y aprendizajes que favorecen conocimientos vivenciados, reflexivos, y críticos, además de creativos.

Por otro lado, autores como Fraser et all (2001), inciden en la importancia de la utilización del movimiento para adquirir el conocimiento de cualidades del sonido, como el ritmo, para vivenciar la música, considerando que «el ritmo es el principio organizador de toda pieza musical». Para otros autores, como Sáenz-López (1997), utilizar el movimiento como medio de comunicación es una de las corrientes actuales de la educación física, ya que históricamente, la danza y la comunicación no verbal se remontan a culturas primitivas, que han ido evolucionando hasta consolidarse en la corriente rítmica, en donde destacan nombres como Delsarte (1811 - 1871), Duncan (1878-1929), Dalcroze (1865-1959) y, posteriormente, Bode (1891-1968) o Jalkanen (1889-1964). Son muchos los profesionales de educación que fundamentan su relevancia en la formación musical de los alumnos y en la relación que mantiene con ella la expresión corporal para lograr un aprendizaje significativo ante los conocimientos musicales, ya que partimos de la idea de que el origen del movimiento está en la música.

La expresión corporal, para Stokoe y Schächter (1886), es de por sí, un lenguaje que integra las área motriz, cognitiva y afectiva, en opinión de Sáenz-López (1997).

Desde nuestro punto de vista, la educación musical y la expresión corporal son medios importantes para desarrollar la creatividad. Para Oriol (2001) y Bolaños (2006), las actividades expresivas, han adquirido cada vez más presencia en la escuela, considerándolas fundamentales para el desarrollo de la actividad creadora de los alumnos y para los procesos de socialización.

El movimiento es una conducta necesaria e imprescindible de todo ser humano, que entendida como vehículo de expresión y comunicación, ayuda a vivenciar significativamente la música, además de fomentar el desarrollo creativo de la persona. Desde nuestro punto de vista, para promover el desarrollo de la creatividad, consideramos que se centra en el desarrollo de la sensibilidad del ritmo, la expresividad, el control corporal, la generación de ideas de movimientos, que favorecen en el alumno un aprendizaje que proporciona experiencias significativas degran valor educativo en el alumnado de Educación Secundaria Obligatoria a través de la expresión corporal.

Siguiendo la opinión de Omecaña y Ruiz (2005), en nuestro estudio, pretendemos desarrollar actividades lúdicas que potencien la creatividad, a través de la creación musical, frente a la reproducción de estereotipos.

En nuestra investigación, siguiendo a autores como Berrade(2010), consideramos que adquieren gran importancia como aspecto fundamental de creatividad o desarrollo creativo de la persona, «la capacidad de iniciativa que ésta puede poseer»; además de «la capacidad de comunicación», según Oriol (2001), que permite la expresión creativa, sobre todo a través de la música.

\section{Modelos de enseñanza}

\section{Modelo de enseñanza tradicional}

El modelo tradicional es el modelo triangular, muy extendido en la didáctica, que postula que la enseñanza es una relación entre tres componentes, que son, el profesor, el alumno y la materia, según Gimeno (1989).

Según este autor, en este modelo de enseñanza el emisor es único y no intercambiable con el receptor, además de que no se preveen relaciones entre los elementos.

La opinión de Waisburd y Erdmenger (2007), por otro lado, señalan que los paradigmas tradicionales promueven una enseñanza con conocimientos y habilidades aisladas, aceptando el conocimiento de forma pasiva, en la que el maestro enseña de forma lógica, concreta e individual a grupos homogéneos, brindando conocimientos especializados y específicos, en los que prevalece el pensamiento lineal, las respuestas correctas y únicas, exámenes para probar el conocimiento, y metas y objetivos determinados por el maestro.

A través de este tipo de enseñanza, es cierto, que se centra la atención en el docente como protagonista y fuente del saber, que es transmitido a los alumnos. La información circula siempre en un único sentido y el aprendizaje se realiza a base de repetir mecánicamente, o usa estrategias nemotécnicas encaminadas al recuerdo reproductivo, como considera Zaragozá (2009). En este sentido, podemos decir que se busca la convergencia entre el profesor y el alumno permanentemente, y que es un modelo de enseñanza formal, directo y cerrado.

El modelo de enseñanza tradicional ha tenido una gran importancia en el ámbito educativo, pero en la actualidad, hay autores que consideran que presenta limitaciones en su puesta en práctica en el aula. En nuestro caso, seguimos la opinión de De la Torre (1998), citado en Gervilla (2003), afirma que la enseñanza tradicional debe dar entrada a nuevas consideraciones de la realidad.

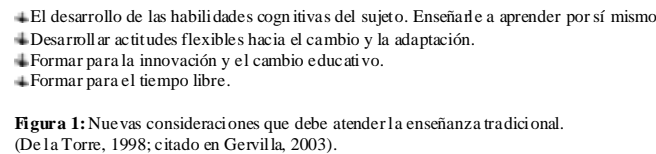

Teniendo en cuenta cómo enseña el profesor a través de una enseñanza tradicional y cómo se genera el aprendizaje del alumno afirmamos que el profesor mediante este modelo de enseñanza, adquiere el papel de emisor. Realmente, es el que protagoniza el proceso de enseñanza aprendizaje, siendo la única fuente de saber, por lo que su función a lo largo del proceso no es intercambiable con la del alumnado, porque éstos se limitan a recibir los contenidos que deben aprender, de una manera precisa, en donde sus experiencias, intereses y expectativas no se tienen en cuenta. El aprendizaje de los alumnos es básicamente receptivo.

En base a lo comentado, el profesor es el único que enseña, y por tanto, el único que proporciona el conocimiento de los contenidos a los alumnos. Por ello, la relación que se establece entre el profesor y el alumno podemos considerarla autoritaria y directiva. 


\section{Modelo de Enseñanza Creativo}

En las últimas décadas, son muchos los teóricos de la educación que apoyan la idea de utilizar una metodología creativa en el proceso de enseñanza-aprendizaje que se genera en las aulas.

Entre los grandes propulsores de la creatividad en la enseñanza se encuentran según Torrance (1977), Marín $(1974,1975)$ y De la Torre (1991); planteando la creatividad como objetivo. En opinión de estos autores, el modelo de enseñanza creativa conlleva en sí mismo un proceso de aprendizaje por parte del alumno, es decir, el alumno debe ser capaz de aprender creativamente, lo cual conlleva un aprendizaje que necesita ser desarrollado. En la revisión de los autores que han venido tratando este tema, como es el caso de Marín(1974), afirma que «una de las primeras condiciones para una pedagogía creativa es la de habituar al alumno a situarse ante el futuro con actitud innovadora», siguiendo a Menchén (1998), opina que debemos «conseguir que el alumno descubra el sentido más puro de la realidad, de tal forma que le permita desplegar su propio estilo de aprendizaje».

Por otra parte, tenemos muy presente que el alumnado aprende haciendo, en contextos reales o significativos, comunicando lo aprendido, siendo consciente del propio proceso de aprendizaje y en interacción con las demás personas. Este planteamiento del aprendizaje contemplado por Garamendi y González (2010), es fundamental en la aplicación de cualquier modelo de enseñanza creativa.

A través de la enseñanza creativa, siguiendo la opinión de Rodríguez Estrada (1993), dotamos a nuestro alumnado de iniciativa, recursos, confianza en sí mismos y en la vida, por lo que serán capaces de enfrentarse a problemas de cualquier índole. Por ello, fomentamos así una educación integral. Atendiendo a la opinión de Torrance (1977), afirma que un niño aprende creativamente «interrogando, inquiriendo, buscando, manipulando, experimentando, hasta en el simple juego». Desde nuestro punto de vista, todo modelo de enseñanza creativa debe desarrollar este tipo de aprendizaje en el alumnado, para que pueda desarrollar su creatividad.

Por otra parte, consideramos importante atender a la opinión de Lowenfeld y Lambert (1985), los cuales afirman que la capacidad creadora del alumnado de Secundaria debe desarrollarse en un programa planificado, contemplando los indicadores de la creatividad como son «la flexibilidad, la fluidez, la originalidad y la facultad de pensar en forma independiente e imaginativa». Desde nuestro punto de vista, el programa que pretendemos llevar a cabo en Educación Secundaria, debe estar planificado, pero abierto a ser modificado en la práctica.

Desde el punto de vista de estos autores y según nuestra opinión, la enseñanza creativa debe centrarse en el modo de pensary de actuar del alumno, porque es la que le va a permitir al alumno enfrentarse a la actividad de manera creativa, aportando sus experiencias, percepciones y descubrimientos, favoreciendo el aprendizaje de habilidades, estrategias de trabajo, actitudes, aptitudes y destrezas, para promover el aprendizaje integral y creativo del alumno. Considerando la opinión de autores como Coll y Espinosa (2006), siguiendo a Runco y Sakamoto (1999), afirman que la creatividad está influida por una amplia serie de experiencias evolutivas, sociales y educativas, y se manifiestan de maneras diferentes, lo cual desde nuestra opinión, enriquece considerablemente el aprendizaje de los alumnos.

Desde nuestro punto de vista y según han apoyado anteriormente muchos autores de gran relevancia, destacamos la importancia y el papel que ejercen en el proceso de enseñanza-aprendizaje el profesor, el alumno y los contenidos a desarrollar, entendiendo así que el profesor debe promover en su alumnado este tipo de aprendizaje a través de diferentes técnicas y estrategias que proporcionen un desarrollo creativo en el alumnado; y el alumnado a su vez, debe estar motivado, estar abierto y ser flexible en su aprendizaje; $y$ los contenidos que se trabajen deben plantearse al alumno de manera atractiva. La atmósfera de trabajo en la clase debe ser abierta y dinámica entre el profesor, el alumnado y los contenidos, generando un enfoque diferente de la información, que potencie la creatividad. Por otra parte, atendemos la opinión de Prieto, López y Ferrándiz (2003), que afirman que el profesor debe fomentar la comunicación, el intercambio de ideas y la ayuda entre los alumnos, para desarrollar una enseñanza creativa. Además de que la forma en que los maestros responden en el aula, tiene una gran influencia sobre la formación de los estudiantes, según López Frías (2004).

Para De la Torre (2003) cuando habla de enseñanza creativa, piensa en «estrategias basadas en el aprendizaje relevante, en el desarrollo de habilidades cognitivas, en una actitud transformadora; en la organización de actividades innovadoras, flexibles, motivantes; en una mediación que tome en consideración la experiencia, la colaboración, la implicación del discente».

Concretando la opinión de este autor sobre la enseñanza creativa según López Frías (2004), fundamentándose en la opinión de De la Torre (1995), comenta que este tipo de enseñanza se caracteriza por cuatro aspectos, es decir, por ser activa, motivadora, dinámica e implicativa.

Por otro lado, estudiando la opinión de Borthwick (1982), considera que en base a los principios planteados por Logan y Logan (1980), la enseñanza creativa es de naturaleza flexible, se caracteriza por los métodos de enseñanza indirecta, es imaginativa, fomenta el uso único de materiales e ideas, favorece la relación, es de naturaleza integradora, refuerza la autodirección, implica autovaloración, y comporta riesgos, pero aporta recompensas.

Para Marín (1989), sin embargo, lo que realmente importa es que este tipo de enseñanza favorezca que cada cual aporte algo personal, valioso e innovador. Por este motivo, lo importante es diseñar actividades que den ocasiones a que afloren aportaciones originales. Según este autor, las tareas rutinarias no pueden desarrollar el aprendizaje creativo delalumno.

Desde nuestro punto de vista, que el alumno adquiera un desarrollo de la creatividad a través de la educación es un requisito fundamental para poder adquirir a lo largo de ella las competencias básicas curriculares. Y tal y como opina Marín (1974), debemos acostumbrar al alumno a las situaciones de carácter problemático, que desde la creatividad, «son muchos los caminos que pueden conducir a una solución y porque normalmente las soluciones suelen ser tan plurales, multifacéticas, como la vida misma». Según este autor, saber acotar los problemas es ya un primer paso en la actitud creadora.

\section{Método}

Desde el punto de vista metodológico, nos encontramos ante una investigación empírica (experimental), basada en la recogida de datos de una realidad educativa, orientada a la mejora de la práctica docente.

\section{Participantes}

El estudio se llevó a cabo en dos centros escolares de la ciudad de Jerez de la Frontera, en la provincia de Cádiz, durante el curso académico 2010/ 2011.

Los centros educativos en los que hemos realizado nuestro estudio son colegios concertados, que proporcionan a sus estudiantes Educación Infantil, Educación Primaria, Educación Secundaria Obligatoria, y en el caso del primer centro al que nos referimos ofrece también Bachillerato. A partir de este momento, nos referiremos a dichos centros como Centro A y Centro B.

En cada uno de los centros en los que desarrollamos nuestra investigación, decidimos que para aumentar la validez interna de ésta, debíamos establecer un grupo control y un grupo experimental sobre el que realizaremos el tratamiento correspondiente al método de la investigación experimental.

La muestra de nuestro estudio la forman cuatro aulas de $1^{\circ} \mathrm{ESO}$, de dos centros educativos de características similares.

El alumnado seleccionado para la experimentación pertenecen a dos grupos A y B, ya establecidos en sus propios centros educativos, contando entre los cuatro grupos con un número total de 115 alumnos.

Esta investigación se desarrolló a lo largo del segundo y tercer trimestre del curso académico 2010/11, con un total de 25 horas de clase, contando semanalmente con 2 horas con cada grupo de investigación (grupo control y grupo experimental). 
Dos de las aulas que hemos tomado como muestra corresponden al Centro A, y con los sub-grupos A1 y A2; y las otras dos, pertenecen al Centro B correspondiéndose con los sub-grupos B1 y B2. Concretando esta relación que mantenemos entre los cuatro sub-grupos que hemos formado, A1 y B1 pertenecen al grupo control, y A2 y B2 al grupo que establecido como experimental.

Los grupos de sujetos participantes en nuestra investigación, están constituidos por alumnos y alumnas. Las edades entre las que se encuentran oscila entre doce, trece, y catorce años en menor proporción.

\section{Instrumentoy procedimiento}

El instrumento de medida que se ha utilizado en nuestro estudio es el Cuestionario CMESO, de 22 ítems, del que se han seleccionado los ítems relativos a la comunicación y al nivel de inventiva, para la presente investigación

El cuestionario fue administrado en el segundo y en el tercer trimestre del curso académico 2010/11, a cada grupo de investigación (grupo control y grupo experimental).

El Cuestionario CMESO, es un instrumento cuantitativo, con el que podemos valorar contenidos cualitativos, como es el desarrollo creativo, elaborado en base a los 11 indicadores de creatividad que establece Marín y De la Torre (2000, 100-109) y las cuatro cualidades correspondientes al sonido. En total este cuestionario cuenta con 22 ítems, y tres opciones de respuesta en cada uno, todas ellas verdaderas. Cada ítem o pregunta del cuestionario contempla 3 opciones de respuesta distintos, que hemos establecido como nivel alto, nivel medio y nivel bajo en relación a cada uno de los indicadores que hemos contemplado.

Vamos a aplicar un diseño experimental, ya que podemos tomar cada uno de los grupos clase ya establecidos en los centros educativos que estén implicados en la investigación. En nuestro caso, trabajaremos con dos grupos experimentales en el Centro A y con otros dos grupos en el Centro B, es decir, con un total de 4 grupos experimentales. De cada uno de los centros que vamos a investigar, tomaremos un grupo como control y otro como experimental. En nuestro caso, al grupo experimental se le aplicará el modelo de enseñanza creativo, y al grupo control no se le someterá a dicho tratamiento, se seguirá enseñando a través del modelo de enseñanza tradicional.

La aplicación del tratamiento en los dos grupos experimentales establecidos en cada Centro Educativo, la realizamos de la siguiente forma. En los grupos A1 (del centro A) y B1 (del centro B), se trabajará el modelo de enseñanza tradicional; y en los grupos A2 (del centro A) y B2 (del centro B), el modelo de enseñanza creativo. En todos los grupos establecidos desarrollaremos la realización de una creación musical.

Las medidas pre-test y pos-test se han realizado simultáneamente en los dos grupos establecidos en cada centro, y la asignación de sujetos a los grupos estaba establecida de antemano por el grupo clase.

Tras la realización del experimento realizamos un estudio cuantitativo basado en la utilizando técnicas estadísticas univariables, a través del análisis comparativo.

\section{Análisis estadístico}

En el análisis de los datos obtenido a lo largo de nuestra investigación, hemos analizado e interpretado los resultados obtenidos a lo largo de nuestro estudio, realizando la comparación por dimensiones de los resultados iniciales obtenidos del Grupo Control y Experimental en el Pre-test, y la comparación por dimensiones de los resultados alcanzados por el Grupo Control y Experimental en el Post-test.

El propósito que hemos perseguido en el análisis de los datos obtenidos en nuestra investigación nos permite describir, analizar e interpretar los resultados. La naturaleza de los datos que hemos obtenido es cuantitativa. El análisis de los dos indicadores evaluados en nuestra investigación se ha realizado mediante el Programa Estadístico SPSS v.11, para analizar los resultados obtenidos, tanto en el pre-test como en el pos-test.

Hemos desarrollado un análisis descriptivo de cada una de las variables objeto de estudio, en el que mostramos la frecuencia, el porcentaje y las comparativas. La escala de medida de las variables que se ha empleado ha sido categórica, para que la prueba estadística se adecue a las características métricas de las variables de nuestra investigación.

\section{Resultados}

Al realizar la comparación entre los resultados obtenidos del GC y del GE en el pre-test sobre los ítems establecidos en el cuestionario CMESO, consideramos una mejoría de los resultados cuando obtenemos en el GC y en el GE del nivel bajo una representación menor en las puntuaciones obtenidas en el GE, a diferencia de los resultados que obtengamos en el GE del nivel medio y alto que deben ser superiores a las obtenidas en el GC para que consideremos que exista mejoría.

En la comparación de los resultados del Grupo Control y Experimental en el Pre-test, hemos obtenido lo siguiente:

En los resultados obtenidos sobre el ítem núm. 15, relativo a Comunicación, en el pre-test al comparar el GC y el GE, correspondiente al indicador núm. 8, podemos observar que en el nivel bajo entre el GC y el GE presenta una puntuación más alta en los resultados del GE, representado por un $3.5 \%$, ya que en el GC cuenta con una representación del 18.3\% y en el GE aumenta al 21.8\%. En el nivel medio, el GC ha obtenido el $45 \%$ de representación y el GE el $36,4 \%$, por lo que se observa una diferencia del $8.6 \%$ entre las puntuaciones, siendo menor la obtenida en el GE. El porcentaje alcanzado en el nivel alto sobre este ítem, cuenta con una mejora en los resultados del 5,1\%, ya que en el GC presenta una representación del 36.7\% y en el GE del 41.8\%.

\begin{tabular}{|c|c|c|c|c|c|}
\hline \multicolumn{2}{|c|}{ APERTURA MENTAL } & Frecuencia & Porcentaje & Porcentaje vá lido & $\begin{array}{l}\text { Porcentaje } \\
\text { acumulado }\end{array}$ \\
\hline \multirow{4}{*}{$\begin{array}{l}\text { Válidos GC } \\
\text { (PRE-TEST) }\end{array}$} & N. bajo & 11 & 18.3 & 18.3 & $\begin{array}{l}18.3 \\
\end{array}$ \\
\hline & N. medio & 27 & 45.0 & 45.0 & 63.3 \\
\hline & N. alt to & 22 & 36.7 & 36.7 & 100 \\
\hline & Total & 60 & 100 & 100 & \\
\hline \multirow{3}{*}{$\begin{array}{l}\text { Válidos GE } \\
\text { (PRE-TEST) }\end{array}$} & N. bajo & 12 & 21.8 & 21.8 & 21.8 \\
\hline & N. medio & 20 & 36.4 & 36.4 & 58.2 \\
\hline & $\begin{array}{l}\text { N. al to } \\
\text { Total }\end{array}$ & $\begin{array}{l}23 \\
55\end{array}$ & $\begin{array}{l}41.8 \\
100\end{array}$ & $\begin{array}{l}41.8 \\
100\end{array}$ & 100 \\
\hline \multicolumn{6}{|c|}{$\begin{array}{l}\text { Tabla 2: Frecuencia del GC y GE en el Pre-test, sobre el indicador núm. } 8 \text { relativo a la "Comunicaciór } \\
\text { Item núm. } 16 \text { del CMESO. }\end{array}$} \\
\hline \multicolumn{2}{|c|}{ COMUNICACIÓN } & Frecuencia & Porcentaje & Porcen taje válido & $\begin{array}{l}\text { Porcentaje } \\
\text { acumul ado }\end{array}$ \\
\hline \multirow{4}{*}{$\begin{array}{l}\text { Válidos GC } \\
\text { (PRE-TEST) }\end{array}$} & N. bajo & 22 & 36.7 & 36.7 & 36.7 \\
\hline & N. medio & 25 & 41.7 & 41.7 & 78.3 \\
\hline & $\mathrm{N}$. alto & 13 & 21.7 & 21.7 & 100 \\
\hline & Total & 60 & 100 & 100 & \\
\hline \multirow{4}{*}{$\begin{array}{c}\text { Válidos GE } \\
\text { (PRE-TEST) }\end{array}$} & N. bajo & 23 & 41.8 & 41.8 & 41.8 \\
\hline & N. medio & 20 & 36.4 & 36.4 & 78.2 \\
\hline & N. alto & 12 & 21.8 & 21.8 & 100 \\
\hline & Total & 55 & 100 & 100 & \\
\hline
\end{tabular}

Podemos destacar que sobre el ítem núm. 15 existe una mejora de los resultados obtenidos en el nivel alto. En el GE el porcentaje obtenido en el nivel alto es mayor al del GC en un 5.1\%. El nivel medio, en cambio, presenta un descenso del 8.6\% entre los resultados obtenidos en el GC y en el GE; $y$ en el nivel bajo existe un aumento en la puntuación obtenida en el GE, con una diferencia del 3.5\% con respecto al GC.

En los resultados obtenidos sobre el ítem núm. 16, relativo a Comunicación, en el pre-test al comparar el GC y el GE, correspondiente al indicador núm. 8, podemos observar que en el nivel bajo entre el GC y el GE presenta un descenso en los resultados del GE de un $5.1 \%$, ya que en el GC cuenta con una representación del 36.7\% y en el GE con el $41.8 \%$. En el nivel medio, el GC tiene el $41.7 \%$ de representación y el GE el 36.4\%, por lo que se observa una diferencia del 5.3\% entre las puntuaciones, siendo menor la obtenida en el GE. El porcentaje alcanzado en el nivel alto sobre este ítem, cuenta con una mejora en los resultados del .1\%, ya que en el GC presenta una representación del 21.7\% y en el GE del 21.8\%.

Sobre el ítem núm. 16, existe una mejora de los resultados obtenidos en el nivel alto. En el GE el porcentaje obtenido en el nivel alto es mayor al del GC en un .1\%. El nivel medio, en cambio, presenta un descenso del 5.3\% entre los resultados obtenidos en el GC y en el GE. El nivel bajo presenta, también una menor representación en la puntuación obtenida en el GE, con una diferencia del 5.1\% con respecto al GC.

En los resultados obtenidos sobre el ítem núm. 21, relativo al Nivel de inventiva, en el pre-test al comparar el GC y el GE, correspondiente 
al indicador núm. 11, podemos observar que en el nivel bajo entre el GC y el GE existe una diferencia de un $5.8 \%$, ya que en el GC cuenta con una representación del $43.3 \%$ y en el GE del $49.1 \%$, siendo superior la obtenida en el GE. En el nivel medio, el GC ha obtenido el 20\% de representación y el GE el 12.7\%, por lo que se observa una diferencia del 7.3\% entre las puntuaciones, siendo menor la obtenida en el GE. El porcentaje alcanzado en el nivel alto sobre este ítem, cuenta con una mejora en los resultados del $1.5 \%$, ya que en el GC presenta una representación del 36.7\% y en el GE del 38.2\%.

\begin{tabular}{|c|c|c|c|c|}
\hline NIVEL DE INVENTIVA & Frecuencia & Porcentaje & Porcentaje válido & $\begin{array}{l}\text { Porcentaje } \\
\text { acumulado }\end{array}$ \\
\hline Válidos GC N. bajo & 26 & 43.3 & 43.3 & 43.3 \\
\hline $\begin{array}{l}\text { Valdos GC N. medio } \\
\text { (PRE-TEST }\end{array}$ & 12 & 20 & 20 & 63.3 \\
\hline$($ PRE-TESI) N. alto & 22 & 36.7 & 36.7 & 100 \\
\hline Total & 60 & 100 & 100 & \\
\hline N. bajo & 27 & 49.1 & 49.1 & 49.1 \\
\hline Válidos GE N. medio & 7 & 12.7 & 12.7 & 61.8 \\
\hline (PRE-TEST) N. alto & 21 & 38.2 & 38.2 & 100 \\
\hline Total & 55 & 100 & 100 & \\
\hline
\end{tabular}

Tabla 4: Frecuencia del GC y GE en el Pre-test, sobre el indicador núm. 11 relativo al "Nivel de inventiva”. Item núm. 22 dencia del

\begin{tabular}{ccccc}
\hline NIVEL DE INVENTIVA & Frecuencia & Porcentaje & Porcentaje válido & $\begin{array}{c}\text { Porcentaje } \\
\text { acumulado }\end{array}$ \\
\hline N. bajo & 19 & 31.7 & 31.7 & 31.7 \\
Válidos GC N. medio & 13 & 21.7 & 21.7 & 53.3 \\
(PRE-TEST) N. alto & 28 & 46.7 & 46.7 & 100 \\
Total & 60 & 100 & 100 & \\
N. bajo & 23 & 41.8 & 41.8 & 41.8 \\
Válidos GE N. medio & 7 & 12.7 & 12.7 & 54.5 \\
(PRE-TEST) N. alto & 25 & 45.5 & 45.5 & 100 \\
Total & 55 & 100 & 100 & \\
\hline
\end{tabular}

En el ítem núm. 21, existe una mejora de los resultados obtenidos en el nivel alto. En el GE el porcentaje obtenido en el nivel alto es mayor al del GC en un 5.8\%. El nivel medio y en nivel bajo, en cambio, presentan un descenso en los resultados de un $7.3 \%$ y un $5.8 \%$ respectivamente.

En los resultados obtenidos sobre el ítem núm. 22, relativo al Nivel de Inventiva, en el pre-test al comparar el GC y el GE, correspondiente al indicador núm. 11, podemos observar que en el nivel bajo entre el GC y el GE existe una diferencia de un $10.1 \%$, ya que en el GC cuenta con una representación del $31.7 \%$ y en el GE del $41.8 \%$, siendo superior la obtenida en el GE. En el nivel medio, el GC ha obtenido el 21.7\% de representación y el GE el 12.7\%, por lo que se observa una diferencia del 9\% entre las puntuaciones, siendo menor la obtenida en el GE. El porcentaje alcanzado en el nivel alto sobre este ítem, cuenta con una mejora en los resultados del 1.2\%, ya que en el GC presenta una representación del $46.7 \%$ y en el GE del $45.5 \%$.

Sobre este ítem, existe un descenso de los resultados obtenidos en los tres niveles establecidos. En el GE el porcentaje obtenido en el nivel bajo es inferior al del GC en un 10.1\%; y en el nivel medio y alto, superior en el GE, con unas diferencias del 9\% y del 1.2\% respectivamente.

En la comparación de los resultados del Grupo Control y Experimental en el Post-test, hemos obtenido lo siguiente:

En los resultados sobre el ítem núm. 15, relativo a Comunicación, entre el GC y el GE en el post-test, correspondiente al indicador núm.

\begin{tabular}{|c|c|c|c|c|c|}
\hline \multicolumn{2}{|c|}{ APERTURA MENTAL } & \multirow{2}{*}{$\begin{array}{c}\text { Frecuencia } \\
13\end{array}$} & \multirow{2}{*}{$\begin{array}{c}\text { Porcentaje } \\
21.7\end{array}$} & \multirow{2}{*}{$\begin{array}{c}\text { Porcentaje válido } \\
21.7\end{array}$} & \multirow{3}{*}{$\begin{array}{c}\text { Porcentaje acumulado } \\
21.7\end{array}$} \\
\hline \multirow{5}{*}{$\begin{array}{l}\text { Válidos GC } \\
\text { (Pos-test) }\end{array}$} & N. bajo & & & & \\
\hline & N. medio & 12 & 20 & 20 & \\
\hline & N. alto & 35 & 58.3 & 58.3 & 100 \\
\hline & Total & 60 & 100 & 100 & \\
\hline & N. bajo & 5 & 9.1 & 9.1 & 9.1 \\
\hline \multirow{3}{*}{$\begin{array}{c}\text { Válidos GE } \\
\text { (Pos-test) }\end{array}$} & N. medio & 8 & 14.5 & 14.5 & 23.6 \\
\hline & N. alto & 42 & 76.4 & 76.4 & 100 \\
\hline & Total & 55 & 100 & 100 & \\
\hline
\end{tabular}

Tabla 6: Frecuencia del GC y GE en el Pos-test, sobre el indicador núm. 8 setivo a Tabla 6: Frecuencia del GC y GE en
"Comunicación". Ittem núm. 16 del CMESO.

\begin{tabular}{|c|c|c|c|c|c|}
\hline \multicolumn{2}{|c|}{ COMUNICACIÓN } & \multirow{2}{*}{$\begin{array}{c}\text { Frecuencia } \\
13\end{array}$} & \multirow{2}{*}{$\begin{array}{c}\text { Porcentaje } \\
21.7\end{array}$} & \multirow{2}{*}{$\begin{array}{c}\text { Porcentaje válido } \\
21.7\end{array}$} & \multirow{2}{*}{$\begin{array}{c}\text { Porcentaje acumulado } \\
21.7\end{array}$} \\
\hline \multirow{5}{*}{$\begin{array}{l}\text { Válidos GC } \\
\text { (Pos-test) }\end{array}$} & N. bajo & & & & \\
\hline & N. medio & 20 & 33.3 & 33.3 & 55 \\
\hline & N. alto & 27 & 45 & 45 & 100 \\
\hline & Total & 60 & 100 & 100 & \\
\hline & N. bajo & 5 & 9.1 & 9.1 & 9.1 \\
\hline \multirow{2}{*}{$\begin{array}{l}\text { Válidos GE } \\
\text { (Pos-test) }\end{array}$} & N. medio & 15 & 27.3 & 27.3 & 36.4 \\
\hline & N. alto & 35 & 63.6 & 63.6 & 100 \\
\hline
\end{tabular}

8, se observa que en el nivel bajo se ha obtenido en el GE un descenso del 12.6\% en los resultados, contando en el GE con una puntuación inferior. El GC de éste nivel cuenta con una representación del 21.7\% y el GE con el 9.1\%. En el nivel medio, entre los resultados del GC y los del GE, existe una diferencia representativa del $5,5 \%$ entre sus puntuaciones, ya que el resultado obtenido en el GC es del 20\% y el del GE del 14.5\%, siendo la del GE la de menor puntuación. En el porcentaje alcanzado en el nivel alto, se puede observar una mejora en los resultados en el GC y el GE del $18.1 \%$, ya que se observa en el GC una representación del $58.3 \%$ y en el GE del $76.4 \%$.

En este ítem, existe una mejora de los resultados obtenidos en el nivel bajo y alto. En el GE el porcentaje obtenido en el nivel bajo es inferior al del GC en un 12.6\%, y en el nivel alto superior en el GE un $18.1 \%$. El nivel medio, en cambio, presenta un descenso del $5.5 \%$ entre los resultados obtenidos en el GC y en el GE.

En los resultados obtenidos sobre el ítem núm. 16, relativo a Сотиnicación, entre el GC y el GE en el post-test, correspondiente al indicador núm. 8, se observa que en el nivel bajo se ha obtenido en el GE un descenso del $12.6 \%$ en los resultados, contando en el GE con una puntuación inferior. El GC de éste nivel cuenta con una representación del $21.7 \%$ y el GE con el 9.1\%. En el nivel medio, entre los resultados del GC y los del GE, existe una diferencia representativa del 6\% entre sus puntuaciones, ya que el resultado obtenido en el GC es del 33.3\% y el del GE del 27.3\%, siendo la del GE la de menor puntuación. En el porcentaje alcanzado en el nivel alto, se puede observar una mejora en los resultados en el GC y el GE del 18.6\%, ya que se observa en el GC una representación del 45\% y en el GE del 63.6\%.

Sobre esteítem, existe una mejora de los resultados obtenidos en el nivel bajo y alto. En el GE el porcentaje obtenido en el nivel bajo es inferior al del GC en un $12.6 \%$, y en el nivel alto superior en el GE un $18.8 \%$. El nivel medio, en cambio, presenta un descenso del $6 \%$ entre los resultados obtenidos en el GC y en el GE.

En los resultados obtenidos sobre el ítem núm. 21, relativo al Nivel de inventiva, entre el GC y el GE en el pos-test, correspondiente al indicador núm. 11, se observa que en el nivel bajo se ha obtenido en el GE un descenso del 26.2\% en los resultados, contando en el GE con una puntuación inferior. El GC de éste nivel cuenta con una representación del 31.7\% y el GE con el 5.5\%. En el nivel medio, entre los resultados del GC y los del GE, existe una diferencia representativa del 6.8\% entre sus puntuaciones, ya que el resultado obtenido en el GC es del 15\% y el del GE del 21.8\%, siendo la del GE la de mayor puntuación. En el porcentaje alcanzado en el nivel alto, se puede observar una mejora en los resultados en el GC y el GE del 19.4\%, ya que se observa en el GC una representación del 53.3\% y en el GE del 72.7\%.

\begin{tabular}{|c|c|c|c|c|c|}
\hline NIVEL DE IN & VENTIVA & Frecuencia & Porcentaje & Porcentaje válido & $\begin{array}{c}\text { Porcentaje acumulado } \\
\end{array}$ \\
\hline \multirow{4}{*}{$\begin{array}{c}\text { Válidos GC } \\
\text { (Pos-test) }\end{array}$} & N. bajo & 19 & 31.7 & 31.7 & 31.7 \\
\hline & N. medio & 9 & 15 & 15 & 46.7 \\
\hline & N. alto & 32 & 53.3 & 53.3 & 100 \\
\hline & Total & 60 & 100 & 100 & \\
\hline \multirow{4}{*}{$\begin{array}{l}\text { Válidos GE } \\
\text { (Pos-test) }\end{array}$} & N. bajo & 3 & 5.5 & 5.5 & 5.5 \\
\hline & $\mathrm{N}$. medio & 12 & 21.8 & 21.8 & 27.3 \\
\hline & N. alto & 40 & 72.7 & 72.7 & 100 \\
\hline & Total & 55 & 100 & 100 & \\
\hline
\end{tabular}

Tabla 8: Frecuencia del GC y GE en el Pos-test, sobre el indicador núm. 11 relativo al “Nivel de inventiva”. tem núm. 22 del CMESO.

\begin{tabular}{cllccc}
\hline \multicolumn{2}{c}{ NIVEL DE INVENTIVA Frecuencia } & Porcentaje & Porcentaje válido & Porcentaje acumulado \\
\hline \multirow{2}{*}{$\begin{array}{c}\text { Válidos GC } \\
\text { (Pos-test) }\end{array}$} & N. bajo & 12 & 20 & 20 & 20 \\
& N. alto & 10 & 16.7 & 16.7 & 36.7 \\
& Total & 38 & 63.3 & 63.3 & 100 \\
& N. bajo & 5 & 100 & 100 & \\
Válidos GE & N. medio & 4 & 9.1 & 9.1 & 9.1 \\
(Pos-test) & N. alto & 46 & 7.3 & 7.3 & 16.4 \\
& Total & 55 & 100 & 83.6 & 100 \\
\hline
\end{tabular}

En este ítem, existe una mejora de los resultados obtenidos en el los tres niveles establecidos. En el GE el porcentaje obtenido en el nivel bajo es inferior al del GC en un 26.2\%. El nivel medio presenta una mejora en el GE del 6.8\%, y el nivel alto una mejora del 19.4\%.

En los resultados obtenidos sobre el ítem núm. 22, relativo al Nivel de inventiva, entre el GC y el GE en el pos-test, correspondiente al 
indicador núm. 11, se observa que en el nivel bajo se ha obtenido en el GE un descenso del $10.9 \%$ en los resultados, contando en el GE con una puntuación inferior. El GC de éste nivel cuenta con una representación del 20\% y el GE con el 9.1\%. En el nivel medio, entre los resultados del GC y los del GE, existe una diferencia representativa del 9.4\% entre sus puntuaciones, ya que el resultado obtenido en el GC es del 16.7\% y el del GE del 7.3\%, siendo la del GE la de menor puntuación. En el porcentaje alcanzado en el nivel alto, se puede observar una mejora en los resultados en el GC y el GE del $20.3 \%$, ya que se observa en el GC una representación del 63.3\% y en el GE del 83.6\%.

Sobre este ítem, existe una mejora de los resultados obtenidos en el nivel bajo y alto. En el GE el porcentaje obtenido en el nivel bajo es inferior al del GC en un 10.9\%, y en el nivel alto es superior en el GE un 20.3\%. El nivel medio, en cambio, presenta un descenso del $9.4 \%$ entre los resultados obtenidos en el GC y en el GE.

\section{Discusión}

No se han encontrado investigaciones con las que podamos contrastar datos significativos relacionados con este estudio para corroborar o contradecir los resultados que hemos obtenido.

Las investigaciones llevadas a cabo sobre creatividad, modelos de enseñanza y expresión corporal en Educación Secundaria Obligatoria, y más concretamente sobre las dimensiones que hemos trabajado en este estudio, no contemplan un análisis concurrente sobre aspectos coincidentes con los nuestros.

No obstante, respecto a la dimensión relativa a la comunicación, encontramos investigaciones como la de Motos (2013), en la que contempla en su práctica, las habilidades de los alumnos para expresar y comunicar con un código específico, para poder progresar en la creación, la expresión y la comunicación, a través de la dramatización; además de presentar una revisión de los instrumentos y técnicas para la evaluación de habilidades de creatividad dramática, haciendo referencia a tests estandarizados y otras alternativas.

Por otra parte, se destaca un estudio realizado por Contreras (2008), en el que trabajó el desarrollo de habilidades creativas con el alumnado de sexto de primaria, haciendo especial énfasis en despertar la imaginación y creatividad de sus alumnos desde la práctica, para que puedan generar ideas novedosas y poder invitarlos a comunicarse a través del uso creativo del cuerpo. Como resultado, el 70\% del alumnado logró un resultado positivo.

\section{Conclusiones}

A partir de los datos analizados, podemos concluir con los resultados obtenidos en el ítem 15 del GC, correspondiente al indicador relativo a Comunicación, en el nivel bajo entre el pre-test y el pos-test presenta una puntuación más alta en los resultados del pos-test, representado por un 3.4\%, siendo mayor la del post-test. En el nivel medio, se observa una diferencia del $25 \%$ entre las puntuaciones, siendo menor la obtenida en el pos-test. El porcentaje alcanzado en el nivel alto, sin embargo, presenta una mejora en los resultados del 21.6\%. Sobre los resultados obtenidos en el ítem núm. 16 del GC, en el nivel bajo entre el pre-test y el pos-test presenta un descenso en los resultados del postest de un 15\%. En el nivel medio, hay una diferencia del $8.4 \%$ entre las puntuaciones, siendo menor la obtenida en el pos-test. El porcentaje alcanzado en el nivel alto sobre este ítem, cuenta con una mejora en los resultados del $23.3 \%$ en el pos-test.

Sobre los resultados obtenidos en el ítem núm. 21 del GC, correspondiente al indicador relativo al Nivel de inventiva, en el nivel bajo entre el pre-test y el post-test existe una diferencia de un $11.6 \%$, siendo inferior la obtenida en el post-test. En el nivel medio, se observa una diferencia del $5 \%$ entre las puntuaciones, siendo menor la obtenida en el post-test. El porcentaje alcanzado en el nivel alto sobre este ítem, cuenta con una mejora en los resultados del $16.6 \%$. Y en los resultados obtenidos sobre el ítem núm. 22 del GC, podemos observar que en el nivel bajo entre el pre-test y el post-test existe una diferencia de un $11.7 \%$, siendo inferior la obtenida en el post-test. En el nivel medio, existe una diferencia del $5 \%$ entre las puntuaciones, siendo menor la obtenida en el posttest. El nivel alto, cuenta con una mejora en los resultados del $16.6 \%$ en el post-test.

En cambio, sobre los resultados obtenidos en el ítem núm. 15 del GE, correspondiente al indicador núm. 8, relativo a Comunicación, podemos observar que en el nivel bajo entre el pre-test y el post-test presenta un descenso en los resultados del post-test de un $12.7 \%$. En el nivel medio, se observa una diferencia del $21.9 \%$ entre las puntuaciones, siendo menor la obtenida en el post-test. El porcentaje alcanzado en el nivel alto sobre este ítem, cuenta con una mejora en los resultados del $34.6 \%$. Y en los resultados obtenidos sobre el ítem núm. 16 del GE, el nivel bajo entre el pre-test y el post-test presenta una mejora en los resultados del pos-test de un 32.7\%. En el nivel medio, se observa una diferencia del 9.1\% entre las puntuaciones, siendo menor la obtenida en el post-test. El porcentaje alcanzado en el nivel alto, cuenta con una mejora en los resultados del $41.8 \%$ en el post-test.

En los resultados obtenidos sobre el ítem núm. 21 del GE, correspondiente al indicador núm. 11, relativo al Nivel de inventiva, podemos observar que en el nivel bajo entre el pre-test y el post-test existe una diferencia de un $43.6 \%$, siendo inferior la obtenida en el post-test. En el nivel medio, se observa una diferencia del 9.1\% entre las puntuaciones, siendo mayor la obtenida en el post-test. El porcentaje alcanzado en el nivel alto sobre este ítem, cuenta con una mejora en los resultados del $34.5 \%$ en el post-test. Y en los resultados obtenidos sobre el ítem núm. 22 del GC, correspondiente al indicador núm. 11, podemos observar que en el nivel bajo entre el pre-test y el post-test existe una diferencia de un 32.7\%, siendo inferior la obtenida en el post-test. En el nivel medio, se observa una diferencia del $5.4 \%$ entre las puntuaciones, siendo menor la obtenida en el post-test. El porcentaje alcanzado en el nivel alto, cuenta con una mejora en los resultados del $38.1 \%$ en el post-test.

\section{Referencias}

Berrade, J. (2010). Estrategias para enseñar y aprender música en el aula: la creación. En Giráldez, A. (coord.). Didáctica de la música. Barcelona: Graó.

Bolaños Bolaños, G. (2006). Educación por medio del movimiento y expresión corporal. (11 edición). Costa Rica: Editorial Universidad Estatal a Distancia San José.

Borthwick, G. (1982). Hacia una educación creativa. Madrid: Editorial Fundamentos.

Coll, \& Espinosa, F.J. (coord.) (2006). Arteterapia: dinámicas entre creación y procesos terapéuticos. Murcia: Universidad de Murcia.

Contreras, M.I. (2008). El desarrollo de la creatividad en sexto grado de nivel primaria. México: Secretaría de Educación Pública. Universidad Pedagógica Nacional.

Cuevas, S. (2011). Expresión artística en Educación Secundaria Obligatoria: Educación musical y movimiento creativo. Actas del IX Congreso Internacional sobre la enseñanza de la Educación Física yel deporte escolar. (pp. 163-169). Úbeda (Jaén).

De la Torre, S. (1991). Evaluación de la creatividad. TAEC: un instrumento de apoyo a la Reforma. Madrid: Escuela Española.

De la Torre, S. (1995). Creatividad aplicada. Recursos para una formación creativa. Madrid: Escuela Española.

De la Torre, S. (2003). Dialogando con la creatividad: de la identificación a la creatividad paradójica. Barcelona: Octaedro.

Fraser, A., Froseth, J.O., \& Weikart, P. (2001). Música y movimiento. Actividades rítmicas en el aula. ( $\left.\mathrm{N}^{\circ} 164\right)$. Barcelona: Editorial Graó.

Garamendi, B., \& González, I. (2010). Innovación educativa en el área de música. En Giráldez, A. (coord.). Música. Investigación, innovación y buenas prácticas. ( $\mathrm{N}^{\circ} 13$, Vol. III). Barcelona: Graó.

Gervilla, Á. (1986). La creatividad en el aula. Actividades para un currículum creativo. Málaga: Ediciones Innovare.

Gervilla, Á. (dir.). (2003). Creatividad aplicada: una apuesta de futuro. Madrid: Dykinson.

Gimeno Sacristán, J. (1989). Teoría de la enseñanza y desarrollo del 
currículo. (7ª Reimp.). Madrid: Anaya.

Kanellopoulos, P.A. (2012). Envisioning Autonomy through Improvising and Composing: Castoriadis visiting creative music education practice. Educational Philosophy and Theory, 44(2), pp. 151-182. Recuperado de http://0-www.scopus.com.fama.us.es/rec o r d / d i s p l a y. u r l ? e i d = 2 - s 2 . 0 $84856865986 \&$ origin=resultslist $\&$ sort $=$ plf $\mathrm{f} \& \mathrm{src}=\mathrm{s} \& \mathrm{st} 1=$ musical + education + and + creativit $\mathrm{y} \& \mathrm{nlo}=\& \mathrm{nl} \mathrm{r}=\& \mathrm{nls}=\& \mathrm{sid}=35 \mathrm{zpKjGR9bjVxZPG0DaSd}$ VL\%3a30\&sot=b\&sdt=b\&sl=114\&s=TITLE-ABS- KEY $\% 28$ musical+education +and + creativity $\% 29+$ AND+SUBJAREA\%28MULT+OR+ARTS+OR+BUS $\mathrm{I}+\mathrm{OR}+\mathrm{DECI}+\mathrm{OR}+\mathrm{ECON}+\mathrm{OR}+\mathrm{PSYC}+\mathrm{OR}+\mathrm{SOCI} \% 29$ \&relpos=0\&relpos $=0 \&$ searchTerm $=$ TITLE-ABS-KEY (musical education and creativity) AND SUBJAREA(MULT OR ARTS OR BUSI OR DECI OR ECON OR PSYC OR SOCI)

Logan, L.M., \& Logan, V.G. (1980). Estrategias para una enseñanza creativa. Barcelona: Oikos-tau.

Lowenfeld, V., \& Lambert Britten, W. (1984). Desarrollo de la capacidad creadora. Madrid: Editorial Kapelusz.

Ley Orgánica 2/2006, de 3 de mayo, de Educación.

López Frías, B.S. (2004). Pensamiento crítico y creativo. (1º Ed.). Sevilla: Ed. Trillas.

Marín Ibáñez, R. (1974). La creatividad en la educación. Buenos Aires: Editorial Kapelusz.

Marín Ibáñez, R.(1974). La creatividad en la educación. BuenosAires: Editorial Kapelusz.

Marín Ibáñez, R. (1975). Técnicas del pensamiento creativo. Valencia: ICE.

Marín Ibáñez, R. (1989). La formación de la creatividad. Lección de la Sesión inaugural del curso 1989-90. Madrid: Universidad Nacional de Educación a Distancia.

Menchén Bellón, F. (1998). Descubrir la creatividad. Desaprender para volver a aprender. Madrid: Ediciones Pirámide.

Motos, T. (2013). Habilidades de dramatización y evaluación de la creatividad dramática. Recuperado de http://www.iacat.com/revis- $\mathrm{t}$ a / r e crearte/recrearte $07 / \mathrm{S}$ e c c i on $3 /$ 3.\%20CD.\%20Habilidades\%20dramaticas\%20y\%20ev\%20.pdf Muñoz, J.R. (2007). La expresión instrumental en el ámbito escolar. En Aróstegui, J.L. et al. La creatividad en la clase de música: componer y tocar. Revista Claves para la innovación educativa, $\mathrm{n}^{\circ}$ 40, pp. 2126. Madrid: Editorial Graó.

Omecaña Cilla, R., \& Ruiz Omecaña, J.V. (2005). Juegos cooperativos yeducación física. ( $3^{\mathrm{a} E d i c i o ́ n) . ~ B a r c e l o n a: ~ E d i t o r i a l ~ P a i d o t r i b o . ~}$

Oriol deAlarcón, N. (2001). Estética y creatividad en la educación ante el nuevo milenio. En La educación artística, clave para el desarrollo de la creatividad. (pp. 9-24). Madrid: Ministerio de Educación, Cultura y Deporte.

Pastor Bustamante, A. (1998). La necesidad de crear. En Marín Ibáñez, R; López-Barajas Zayas, E. \& Martín González, M.T. (coords.). Creatividad polivalente. Actas y Congresos (pp. 119-120). Madrid: Universidad Nacional de Educación a Distancia.

Prieto Sánchez, M.D, López Martínez, O., \& Ferrándiz García, C. (2003). La creatividad en el contexto escolar. Estrategias para favorecerla. Madrid: Pirámide.

Real Decreto 1631/2006, de 29 de diciembre, es donde se establecen las enseñanzas mínimas correspondientes a la Educación Secundaria Obligatoria, en relación a uno de los objetivos de esta etapa.

Rivas Navarro, M. (2008). Procesos cognitivos y aprendizaje significativo. Madrid: Comunidad de Madrid, Subdirección General de Inspección Educativa.

Rodríguez Estrada, M.(1991). Creatividad en la investigación científica. México: Editorial Trillas.

Sáenz-López Boñuel, P. (1997). La educación física y su didáctica. Manual para el profesor. ( $2^{\mathrm{a}}$ Ed.). Ed. Wanceulen.

Torrance, E.P.(1977). Educacióny capacidad creativa. Madrid: Marova. Waisburd, G \& Erdmenger, E. (2007). El poder de la música en el aprendizaje. Cómo lograr un aprendizaje acelerado y creativo. Sevilla: Editorial Mad.

Zaragozá, J.L. (2009). Didáctica de la música en la educación secundaria. Revista Eufonía, nº 265. Barcelona: Editorial Graó.

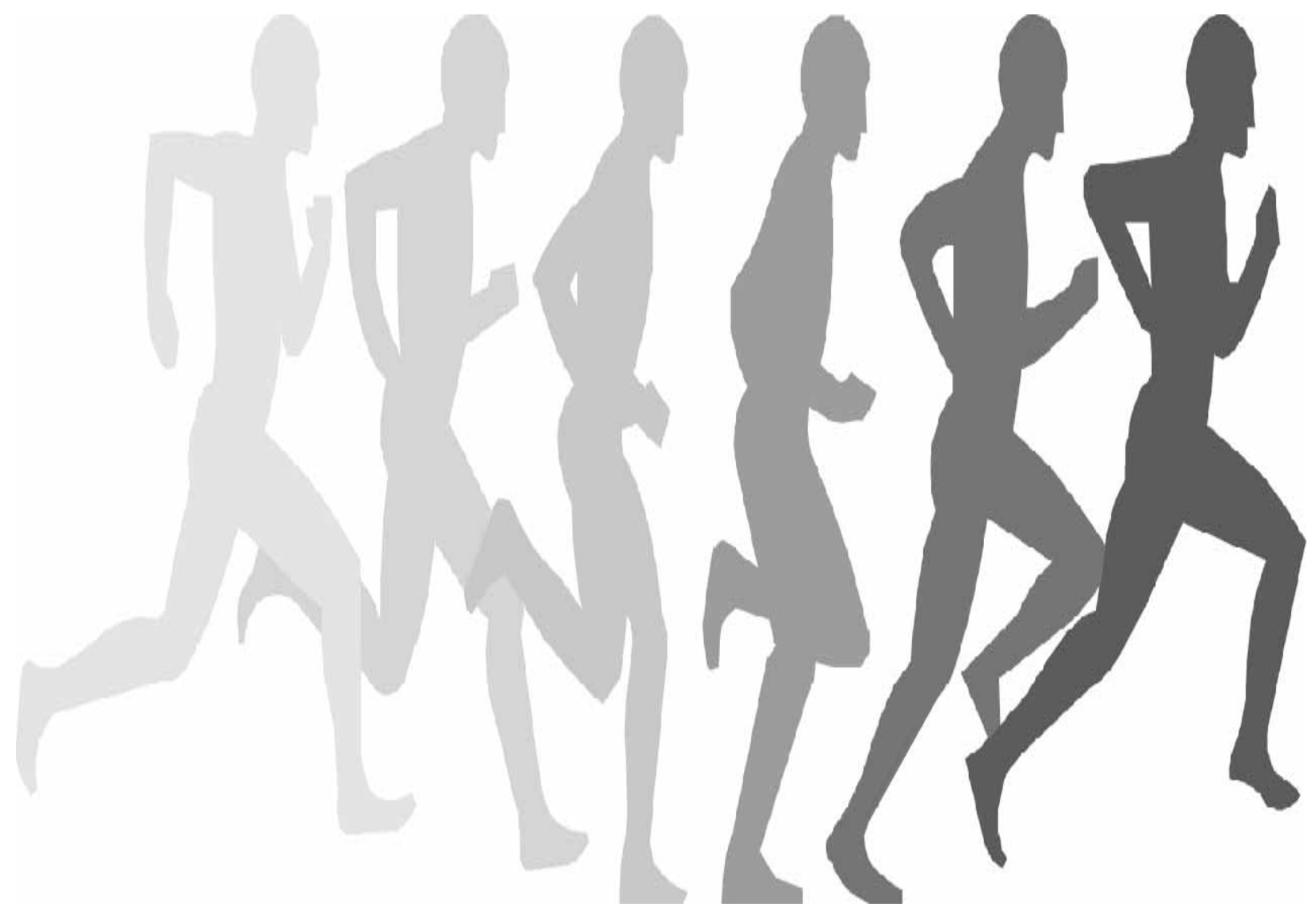

